Revista Venezolana de Gerencia (RVG)

Año 21. No 75, 2016, 361 - 364

Universidad del Zulia (LUZ) • ISSN 1315-9984

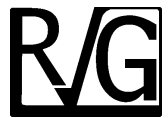

\title{
Editorial
}

\section{Celebrando el vigésimo aniversario de la RVG}

La Revista Venezolana de Gerencia desde el año 1996 en forma ininterrumpida, ha sido uno de los principales medios de difusión académica de la Facultad de Ciencias Económicas y Sociales de la Universidad del Zulia, los resultados y productos de las investigaciones científicas que se publican en sus páginas responden a las necesidades y prioridades de un colectivo de las Ciencias Sociales, con el propósito de difundir los avances de la ciencia, tecnología y la innovación en el ámbito nacional, regional e internacional.

Durante estos veinte años la Revista Venezolana de Gerencia se ha convertido en un referente obligatorio vinculado a los estudios de las Ciencias Sociales, Ilegando a transformarse en una de las grandes realizaciones académicas para la universidad por la productividad científica. Igualmente se destaca el esfuerzo colectivo del personal involucrado y el significado que tiene para nuestra Facultad construir una obra tan perdurable y valiosa como lo es la RVG. Hoy se ve recompensada por una serie de reconocimientos que demuestran un trabajo constante y potenciado con los aportes de los investigadores. Como Directora del Centro de Estudios de la Empresa, destino unas palabras para recalcar la importancia de esta publicación y celebrar el Vigésimo Aniversario dando cuenta de reconocimientos y avances.

Como primera sección se plantea las Perspectivas Editoriales en América Latina, en el trabajo titulado "La Difusión del Conocimiento Contrahegemónico en Venezuela: El Reto de las Revistas Científicas", las investigadoras Ochoa Henríquez, Haydee y Boscán, Elizabeth, del Centro de Estudios de la Empresa-Facultad de Ciencias Económicas y Sociales de la Universidad del Zulia. Expresan que en el marco de un proceso de transformación que busca construir un sistema contrahegemónico al capitalismo, se producen en Venezuela desde fines de los noventa cambios significativos en casi todas las dimensiones de la vida, como parte de los cuales se promueve la producción de conocimiento alternativo al hegemónico alineado a la llamada corriente principal de la ciencia, el cual predomina en la mayoría de las revistas editadas en el país. De igual manera revelan que en el contexto de una dominación de la corriente principal de la ciencia en las revistas latinoamericanas, de lo cual no escapa Venezuela, el gobierno promueve la producción de conocimiento contrahegemónico con poca presencia en las revistas del país, lo cual ha estado acompañado de políticas públicas incrementales que no impactan la necesidad de revertir esta situación, por el contrario recientemente tiende a formularse una política pública para las revistas nacionales que reproduce la corriente principal de la ciencia. 
“Gestión de Núcleos Investigativos: Caso de la Universidad de Antofagasta de Chile", es el trabajo presentado por los investigadores Ganga Contreras, Francisco, Villegas Villegas, Francisco, Uriola López, Kattherinne y Tapia Henríquez, Mario, del Departamento de Ciencias del Desarrollo de la Universidad de Los Lagos-Chile y de la Universidad de Barcelona. Su reflexión versa sobre la producción de nuevos saberes es una de las funciones cardinales de toda universidad compleja o que aspire decididamente a tal condición; por lo mismo, estas entidades educativas deben ser capaces de desarrollar estrategias tendientes a aportar en esta perspectiva. Considerando este breve exordio contextual, este trabajo tiene como finalidad primordial exponer los primeros avances respecto de la experiencia en la gestión de los núcleos investigativos en docencia universitaria y su efecto en la gestación de nuevo conocimiento, en una universidad del Estado de Chile.

La segunda sección En la Mira: Política y Gestión Pública Local, inicia con el artículo titulado La Teoría Fundamentada como Herramienta Metodológica para el Estudio de la Gestión Pública Local, de Vegas Meléndez, Hilarion de la Cátedra de Gerencia Pública, Facultad de Ciencias Económicas y Sociales de la Universidad de Carabobo. Refiere que el objetivo principal del trabajo es mostrar de manera sucinta la importancia que tiene la configuración metodológica en el abordaje de estudios relacionados directamente con el tema de la gestión pública local, en la que se busca destacar la importancia que tiene el develar la capacidad de cátedra que tiene la localidad para aprender de sí misma, recomendando establecer una estructura que permita revelar elementos pertinentes y de calidad a través de fuentes primarias, constituidas en los espacios en que se estudia, así como de sus actores sociales.

La Percepción de Habitantes del Norte de Santander sobre Limitaciones del Buen Gobierno, de los Investigadores Albornoz Arias, Neida y Mazuera Arias, Rina, de la Universidad Simón Bolívar, Cúcuta-Colombia. Analizan la percepción de habitantes del Norte de Santander (Colombia) sobre limitaciones del buen gobierno. Se consideran dos elementos del nuevo paradigma de gestión pública, propuestos por el Fondo de las Naciones Unidas para el Desarrollo y el Banco Mundial: ausencia de corrupción y confianza en las instituciones públicas (de todas las ramas del poder, en los ámbitos nacional, departamental y municipal). La percepción de los ciudadanos se obtuvo a través de la aplicación de una encuesta a una muestra para poblaciones finitas de 2.398 personas con edades entre 18 y 69 años, en los cuarenta municipios del Departamento. Existe una marcada percepción de corrupción y desconfianza en las instituciones públicas del Norte de Santander. Esto incide en la credibilidad ciudadana y trae consigo carencias para la existencia de un buen gobierno.

Los autores Valdez Zepeda, Andrés, Viramontes Saldivar, Job y Finol, Rita, adscritos al Centro Universitario de Ciencias Económico Administrativas (CUCEA) de la Universidad de Guadalajara-México y al Centro de Estudios de la Empresa de la Universidad del Zulia, presentan el trabajo Campañas Electorales de Estructuras: Las Claves del Éxito en la Política Electoral. El propósito de esta investigación es explorar las campañas electorales de estructuras: las claves del éxito en la política electoral. La metodología es descriptiva de campo. El resultado de una elección popular, bajo un sistema político de cuño democrático, es de carácter 
multifactorial, especificamente en el resultado de toda elección, inciden muchos factores, desde el perfil del candidato, las estrategias articuladas, los temas de la campaña, los recursos invertidos y hasta el contexto y la circunstancia de la elección, entre otros. Sin embargo, para ganar una elección popular, también se requiere construir y dinamizar diferentes estructuras (políticas, económicas y sociales), mismas que se convierten en maquinarias políticas poderosas que ayudan a persuadir, organizar y movilizar a los votantes. Se concluye que las estructuras juegan un papel muy importante en los resultados de una determinada elección popular, de tal manera que se puede asegurar que el resultado de una elección democrática, se define a nivel de estructuras, dependiendo del tipo de estructura que se logre edificar y dinamizar por parte de los candidatos y/o sus partidos políticos.

\section{El artículo sobre Análisis de la Calidad de Vida en el Cantón Déleg, Provincia del} Cañar-Ecuador, de los autores Jaimes, Edgar; Orellana, René; Reiban, Miriam y González, Juan, de la Universidad del Zulia y de la UCACUE, presenta como objetivo analizar la calidad de vida en los sectores Bayandel, Chini, Déleg y Nueva Alianza, en el Cantón Déleg, Provincia del Cañar, Ecuador. La investigación fue descriptiva, participativa y no probabilística. Se valoraron los factores, variables y subvariables indicadoras implícitas en la calidad de vida de estas comunidades que, en los sectores medio y alto de la subcuenca, es buena ya que el valor promedio del índice de calidad de vida se ubicó en el rango de 8.769 - 16.384.

En la sección Trimestre, los investigadores Tello Castrillón, Carlos y Rodríguez Córdoba, María, de la Universidad Nacional de Colombia, abordan el estudio intitulado Fundamentos Éticos Disciplinares de la Responsabilidad Social Organizacional expresando que ha tenido un sesgo marcado hacia los negocios. Así, la Responsabilidad Social Empresarial es instrumentalizada y se asemeja a una moda de gestión que, por tanto, luego puede ser descartable. Ante ello, el presente artículo asume el concepto más amplio de Responsabilidad Social Organizacional y se propone como objetivo plantear sus relaciones con lo moral. Esto es, reconocer la Responsabilidad Social como resultado ineludible de los fundamentos mismos de la conducta humana. Para tal fin, este escrito se divide en cuatro partes: el concepto de Responsabilidad Social Organizacional, sus fundamentos éticos, su relación con los valores y la ganancia privada y su dimensión pública.

Identidad organizacional de los mandos medios en equilibrio liminal, del investigador Gonzales Miranda, Diego, de la Universidad EAFIT, Colombia, presenta los resultados de una investigación que analizó el proceso de construcción de la identidad organizacional de los mandos medios en una organización colombiana. La problemática se circunscribe a la desestabilización de la identidad como algo dado y relativamente seguro, y un creciente interés por considerarla como objeto y medio de los esfuerzos de regulación por parte de la Administración. La investigación de carácter cualitativo se realizó bajo la modalidad de estudio de caso en la compañía, Comercial Nutresa S.A.S. de Medellín, Colombia. Desde la perspectiva crítica y pluridiciplinaria de los Estudios Organizacionales se propuso definir la identidad organizacional como un diálogo entre la organización y los mandos medios. Los hallazgos que componen el proceso de construcción identitario de los mandos medios fueron: el reconocimiento, la trascendencia y la seguridad. Al analizar sus relaciones y significados para los mandos medios, se puede afirmar que la identidad 
organizacional es un proceso liminal en donde la conjunción de los factores que intervienen en el proceso, configuran un espacio no-estructural en donde el cambio, la resistencia y la constante significación caracterizan la identidad organizacional de los mandos medios de la organización estudiada.

Selección de Proveedores en la minería de oro con lógica difusa, de los autores Gómez, Rodrigo, Cano, José y Campo, Emiro, de la Universidad de Medellín. El artículo tiene como objetivo describir una metodología de evaluación y selección de proveedores basada en un sistema de inferencia difusa (FIS) para el sector minería del oro. Con base en una revisión de la literatura, se desarrolla un sistema difuso conformado por la caracterización del proceso de compras, variables de entrada y salida, funciones de pertenencia, reglas difusas, métodos de agregación y defusificación, e incluye un modelo de ponderación de factores para la generación de reglas difusas. EI FIS propuesto se modela en Matlab®y se valida en una compañía de minería de oro ubicada en el distrito minero de Ataco-Payandé, Colombia. Con los resultados se encuentra que el FIS apoya el proceso de toma decisiones y aumenta las capacidades para la evaluación y selección de proveedores al utilizar modelos cuantitativos, variables lingüísticas y reglas difusas que involucran incertidumbre y ambigüedad. De esta manera, se contribuye al mejoramiento de empresas mineras, donde el proceso de compras y la gestión de proveedores son componentes críticos del sistema logístico minero.

De los investigadores Herrera Vidal, Germán y Herrera Vega, Juan, son docentes investigadores del Grupo de Investigación CIPTEC. Fundación Universitaria Tecnológico Comfenalco-Colombia, el trabajo Modelo de referencia operacional aplicado a una empresa de servicios de mantenimiento refiere que el éxito en las empresas depende de que sus procesos se encuentren alineados e integrados, permitiendo gestionar efectivamente la cadena de suministros. El objetivo del presente artículo es analizar un modelo de referencia operacional, encaminado a determinar oportunidades de mejora e identificar mejores prácticas. Por esto, la investigación es de tipo descriptiva, basada en la metodología planteada por el modelo, mediante el análisis de cinco procesos básicos como planeación, aprovisionamiento, producción, distribución y devoluciones. Se revisó o la literatura, siendo el principal modelo de referencia encontrado el desarrollado por el consejo de cadena de suministros. Se desarrolló la caracterización y análisis de los procesos logísticos y por último la evaluación de las fallas en la ejecución de las actividades.

Finalmente, Wendolin Suárez Amaya, miembro del Comité Editorial de nuestra Revista, recopila relatos, disertaciones y comentarios más resaltantes de las actividades realizadas en el marco de Vigésimo aniversario de la Revista Venezolana de Gerencia, publicación que se constituye en orgullo de nuestra Facultad y de la llustre Universidad del Zulia.

Confiados de que esta edición será un gran aporte al conocimiento generado en las ciencias sociales, y especialmente en la gerencia.

\author{
Mgs. Yorberth Montes de Oca Rojas \\ Directora del Centro de Estudios \\ de la Empresa
}

